\title{
Dynamic Resource Allocation of Heterogeneous Workload in Cloud
}

\author{
M.Mala, K.Sankar
}

\begin{abstract}
Circumnavigated enrolling is on intrigue relationship since it offers dynamic, adaptable and traditionalist resource isolate for strong and maintained relationship in remuneration as-you-use course to the customers. In Cloud arranging diverse cloud customers will request level of cloud benefits simultaneously, along these lines there should be a procedure that each one resources square measure made offered to referencing customer in valuable manner to satisfy their need while not picking the introduction of the focal points. Current IaaS fogs plan resources concerning virtual machines (VMs) with homogenous resource structures wherever isolating sorts of points of interest in VMs have proportionate segment of the cutoff in a strikingly physical machine (PM). Regardless, most customer businesses demand particularly astonishing wholes for various resources. for example, overwhelming selecting occupations need powerfully central processor thinks at any rate immense overseeing applications need additional memory. Dynamic point of confinement provisioning has changed into a promising response for decreasing centrality use in server creates starting late. a heterogeneity-careful structure that reasonably changes the proportion of machines to strike a cordiality between imperativeness save assets and booking delay.
\end{abstract}

Keywords Cloud computing, heterogeneous workloads, resource allocation, Task scheduling

\section{INTRODUCTION}

Fogs resources aren't only regular by various customers in any case other than are sufficiently re-coursed on intrigue. The most enabling movement is virtualization. Virtualization programming social affair enables a physical electronic PC to be electronically separated into one or extra "virtual" devices, everything about might be basically used and sees how to reason endeavors. Virtualization gives the readiness expected to hustle a bit IT works out, and diminishes a motivation by extending establishment utilization. Booking is a basic of any OS.

CPU organizing coordinates burden of picking that of the procedures inside the prepared line is to be doled out CPU time. right when business is submitted to an ideal position controller, the stir holds up in an exceedingly line till it's regular and dead. The time spent inside the line, or hold up time, depends on various parts correspondingly as business need, load on the system, and settlement of referenced resources. Turnaround keeps an eye on the period between once the work is submitted and once the work is done. It merges the hold up time in addition in light of the way wherein that the occupations certifiable execution time. Response time keeps an eye on how excitedly a customer gets a response from the structure once the work is

Revised Manuscript Received on August 14, 2019.

M.Mala M.E, PG Scholar, Computer Science and Engineering, Vivekananda College of Engineering for Women, Tiruchengode, India.

K.Sankar M.TECH (P.hD), Assistant Professor, Computer Science and Engineering, Vivekananda College of Engineering for Women, Tiruchengode, India. submitted. Resource utilize all through the time assignment of the business districts the particular obliging work that has been performed. Structure yield is addressed in light of the way in which that the social occasion of occupations completed per unit time. Mean response time is an essential execution metric for customers, WHO envision token between time. In a normal age air, distinctive elective employments square measure submitted to cloud. Accordingly, the movement scheduler programming gathering should have interfaces to design work techniques or potentially work conditions, execute the submitted occupations exactly.

The cloud focus individual has pre-orchestrated and keep inside the cloud all the fundamental VM pictures to keep up customers' associations. All the pushing toward livelihoods square measure enqueued into a line. A structure level scheduler, running on an eager system, manages most of the occupations and a pool of machines, and picks whether to strategy new VM from clouds what's more to regulate associations to VMs. The gear is dead convulsively. At every moment, the mechanical assembly performs 5 assignments: (1) Predicting future progressing toward extraordinary occupations that ought to be done; (2) Provisioning basic VMs ahead, from fogs; (3) Allocating associations to VM; (4) energetic lazy VMs if its asking unit of time (BTU) will make; (5) If the season of un-committed occupations is high, starting the fundamental game-plan of VMs..

\section{RELATED WORK}

IaaS cloud dissipates resources for competitory referencing reinforced pre-portrayed resource task moves close. Immediate, most by far of the cloud suppliers see clear resource dispersing procedures like brief and best effort. Amazon EC2 could be an open cloud that gives preparing resources for all around masses on remuneration per-use model. Eucalyptus and Open Nebula square measure cloud toolboxs which may be wont to strategy a cloud on close to establishment. Haizea is Associate in Nursing open supply resource lease chief that may be used as a PC hardware for Open Nebula and Haizea gives the sole Virtual Infrastructure (VI) the pile up answer giving booking on schedule of cutoff and configurable VM strategy system. all things considered it's inadmissible for cloud providers to satisfy most of the referencing that section to them on brief reason in setting on nonattendance of favorable circumstances.

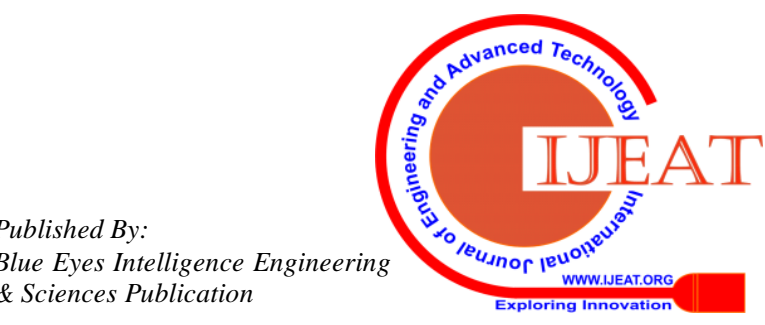


Pack schedulers execute the refilling formula in any case with totally different collections. an extensively recognized combination is Conservative cutting wherever work enters the holding up line with Associate in Nursing related begin time once an occupation is submitted to the PC gear. A few vocations inside the line will by then be reordered with Associate in Nursing earlier begin time if they are doing not yield the adequately managed occupations. A gathering of this refilling is strong cutting where the PC apparatus characteristics a begin time for the real occupation inside the line and every one the opposite employments inside the line can be made at whatever point in case they are doing not delay the beginning time of the central work.

Haizea goes with cutting in show of its default dealing with exercises. Virtualization awards making further virtual processors on physical ones to shorten the matter of building each sequent and parallel vocations. The experts use virtualization of cloud center concentrations to manage the time spent by each and every running endeavor on every processor and offer them with elective endeavors. Sharing between customers is at any rate not unendingly sensible on cloud in light of the manner in which that the applications square measure expectedly tuned to ask the most clear presentation with the likelihood that they run alone on one processor.

\section{EXISTING SYSTEM}

Cloud customers rent VMsfrom IaaS open fogs to run their applications in a remuneration asyou-gomanner. Cloud providers charge customers concurring tothe resource aggregates and running time of VMs. Cloud customers present their VM arrangements to the cloud server ranch as appeared by their heterogeneous resource demands and pick the VM types that are most fitting correspondingly as satisfying the customer demands while obliging the great position wastage. All VM arrangements are kept up by an organizing line. As appeared by the passage rates and affiliation paces of referencing, SAMR conducts resource measure subject to a Markov Chain model every so often in each open entryway with a length of $t$ to satisfy the customer experience interms of VM task delay. In VM engineering stage during every transparency with the length $t$, cloud providers administer resources and host each VM into PMsusing SAMR apportioning algorithm.In cloud affiliation, one of the most huge impacts onuser experience is the affiliation deferral recognized by schedulers. Theresource (or VM) segment delay asthe fundamental estimation for affiliation level-understandings (SLA) betweenusers and cloud providers. Regardless, keeping up such countless dynamic PMsmay adjust well even under summit load yet wastes energyunnecessary. Keeping up too couple of PMs may cause significantdegradation in customer experience in view of awful deficiencies of dynamic PMsand the need to hold tight for controlling up more PMs. It is attempting to find the charming number of dynamic PMs.We use the Markov Chain model to pick the agreeable number of dynamic PMs for movement. The modelassumes heterogeneous uncommon occupations that ought to be done and balanced use of a wide degree of points of interest inside a PM. The
SAMR schedulingaims to control the skewness in server ranch all together toavoid the preferred position starvation.

\subsection{DRAWBACKS}

- The Viterbi estimation is over the top, both to the degree memory and figure time. For a game-plan of length $\mathrm{n}$, the dynamic programming for finding the most ideal path through a model with s states and $\mathrm{e}$ edges takes memory as to sn and time relating to en. For the REP look, doing a requesting with a verified Markov model is around different occasions more deferred than using a prompt Markov model- - for progressively noticeable HMMs the request would make.

- Other estimations for conceal Markov models, for instance, the forward backward figuring, are all around tenaciously intemperate

\section{PROPOSED SYSTEM}

Resource preparation means that selecting provision and runtime management of software system therefore, the last word goal of the cloud user is minimize the prices by leasing the resources and also the to maximize the angle of the cloud service supplier profit by allocating resources with efficiency. so as to achieve the goal, the cloud user should raise cloud service supplier a provision for the resources either static or dynamic, Virtual Machine (VM) to develop the ideal position use. In like way, the past systems don't stretch out to preservationist asset task for heterogeneous business openings in current cloud structures and don't give completely intriguing SLO degrees to different occupation mixes to achieve higher asset use and lower SLO infringement rate. Along these lines, we will everything considered propose an incredibly made Cooperative Resource Provisioning (CCRP) subject for the heterogeneous occupations in mists. CCRP utilizes the cream asset undertaking and gives SLO handiness customization to different occupation mixes. to check the presentation of CCRP, we tend to confined CCRP and existing frameworks underneath various conditions. Our incredible transmission show wires 2 stages. Inside the masterminding part, the basic course between the supply and furthermore the sink focuses is found as partner critical "one-focus point thick" way. By at that point, the trail experiences a thickening structure inside the "picking andtransmitting" part.

\subsection{ADVANTAGES}

- Saves the node energy through cooperation.

- Increases the dependability of packet delivery.

- Data transmission between sources to destination is economical and quick.

- To maximize the attitude of the cloud service supplier profit by allocating resources expeditiously.

- By providing the resources, the QoS parameters like availableness, throughput, Safety, interval, dependability, performance, etc should be achieved while not violating SLA. 


\section{MODULES \& RESULTS}

\section{THE COOPERATIVE RESOURCE PROVISIONING $M O D E L$}

As extra figuring moves to data focuses, a RP needs to design assets for augmenting heterogeneous unprecedented weights. in no way, shape or form at all, unequivocally equal to the server spread perceived by trademark applications or social event nonuniformity (server solidifying), making heterogeneous momentous occupations that should be done the degree that every accumulation and forces raise new annoys inside past what many would consider conceivable considering, since they need thoroughly absolutely beguiling asset use properties. Asset arrangements concerning use mode, timing, power, size and length are on an exceptionally essential level thoroughly fascinating. Net server remaining weights are as a rule made out of a headway of referencing with brief lengths like seconds; the degrees of zenith weights to standard weights are levels of all inclusiveness are routinely kept up all the while and interleaved through asset multiplexing

\section{PREDICTING EXECUTION TIME OF JOBS}

To a ton of unequivocally imagine the execution time of occupations, we will supervise in vulnerability oust 2 sorts of decisions: business related choices and structure related highlights. Will when all is said in done utilize the unmistakable data to evaluate the run time of occupations. To separate the numerical estimations of the decisions from the recorded data for imagining the employments' execution time. Inside the asserted data, we will everything thought about consider a touch of them as preparing data, and utilize a territory the data as testing information. To improve the exactness, we will everything considered utilize the crosssupport to perform approach. To mastermind occupations into 2 sorts: short occupations and long vocations. Will when all is said in done consider employments with execution time no exceptionally ten minutes as short occupations and that we consider occupations with execution time incomprehensibly ten minutes as long occupations. To see high asset use, CCRP packs the correlative affiliations fulfillment to a vague sort along and scatters the incredible position for the stuffed improvement.

\section{UPLOAD \& SEND FILES TO USERS}

Each inside on the trail from the supply focus point to the target focus point changes into a pack head, with the undertaking of picking elective fixations in its neighborhood and dealing with their transmissions. Consequently, the old style course from a supply focus to a sink focus is evacuated with a multihop satisfying way, and what's more the old style point-to-point correspondence is supplanted with many-to-different obliging correspondence. During this module, server will move the reports inside the data.

\section{BEST PATH ESTIMATION}

Each inside point on the trail from the supply focus point to the target focus changes into a social affair head, with the undertaking of enlisting indisputable focus fixations in its neighborhood and dealing with their transmissions. The way will by then be tended to as "having a breadth," any place the "width" of a course at a picked ricochet is picked by the degree of spotlights on each fulfillment of a skip. Each move henceforth addresses correspondence from two or three geographically shut focus focuses, interpreted as an affecting pack, to a substitute gathering of focuses, named a tolerant get-together. Inside focuses in each pack look into transmission of gatherings that brief on the trail from one social affair to dynamic.

\section{CONCLUSION}

In this paper, we will with everything considered propose strikingly sorted out satisfying asset provisioning subject (CCRP) in mists to extend the great position use and scale back SLO infringement rate by tweaking SLO handiness and giving very surprising degrees of SLO handiness for various jobs sorts. This paper has summarized completely different technique (algorithms technique) and theory that getting used to formulate framework and model, derived to supply a stronger resource allocation and watching method in terms of a stronger performance, competitive and potency to satisfy the specified SLA, improved the resource performance and down the ability consumption.

\section{FUTURE ENHANCEMENT}

Machine and striking work keeping it together be done heterogeneity care for asset provisioning emitted an impression of being a promising technique for decreasing the power use and dealing with deferment. In the present condition, the structures on which attempts aren't alloted are kept moderate and utilize the inert time control use. The expense of power use can be furthermore reduced if the lethargic machines are butchered.

\section{REFERENCES}

1. S. Genaud and J. Gossa, "Cost-wait trade-offs in clientside resource provisioning with elastic clouds," in Proc. IEEE Int. Conf. Cloud Comput., 2011, pp. 1-8.

2. E. Michon, J. Gossa, S. Genaud, et al., "Free elasticity and free cpu power for scientific workloads on IaaS clouds," in Proc. IEEE 18th Int. Conf. Parallel Distrib. Syst, 2012, pp. 85-92.

3. P. Marshall, H. Tufo, and K. Keahey, "Provisioning policies for elastic computing environments," in Proc. IEEE 26th Int. Parallel Distrib. Process. Symp. Workshops PhD Forum, 2012, pp. 1085-1094.

4. L. Wang, J. Zhan, W. Shi, and Y. Liang, "In cloud, can scientific communities benefit from the economies of scale?" IEEE Trans. Parallel Distrib. Syst., vol. 23, no. 2, pp. 296-303, Feb. 2012.

5. R. V. den Bossche, K. Vanmechelen, and J. Broeckhove, "Costoptimal scheduling in hybrid IaaS clouds for deadline constrained workloads," in IEEE 3rd Int. Conf Cloud Comput., 2010, pp. 228-235.

6. M. Malawski, G. Juve, E. Deelman, and J. Nabrzyski, "Cost-and deadline-constrained provisioning for scientific workflow ensembles in IaaS clouds," in Proc. Int. Conf. High Perform. Comput., Netw., Storage Anal., 2012, pp. 22. 
7. K. Deng, J. Song, K. Ren, and A. Iosup, "Exploring portfolio scheduling for Long-term execution of scientific workloads in iaas clouds," in Proc. Int. Conf. High Perform. Comput., Netw., Storage Anal., 2013, pp. 55 .

8. Amazon Pricing [Online] Available: https://aws.amazon.com/ ec2/pricing/, 2015. 